\title{
Controlling continuous bioreactor via nonlinear feedback: modelling and simulations approach
}

\author{
R. AGUILAR-LÓPEZ ${ }^{1 *}$ and I. NERIA-GONZÁLEZ ${ }^{2}$ \\ ${ }^{1}$ Department of Biotechnology and Bioengineering, Centro de Investigación y Estudios, Avanzados del Instituto Politécnico Nacional, \\ Av. Instituto Politécnico Nacional No. 2508, Colonia San Pedro Zacatenco, 07360, Ciudad de México D.F., Méxio \\ ${ }^{2}$ Chemical and Biochemical Engineering Division. Tecnológico de Estudios Superiores de Ecatepec. Av. Tecnologico S/N, \\ Colonia Valle de Anahuac, 55210, Ecatepec de Morelos, Edo. Mexico, Mexico
}

\begin{abstract}
The aim of this work is to present a class of nonlinear controller with an exponential-type feedback in order to regulate the sulfate mass concentration via the input flow in a continuous stirred tank bioreactor of an anaerobic sulfate-reducing process. The corresponding kinetic terms in the bioreactor's modeling are modeled by unstructured modeling approach, which was experimentally corroborated. A sketch of proof of the closed-loop stability of the considered system is done under the framework of Lyapunov theory. Numerical experiments are conducted to show the performance of the proposed methodology in comparison with a well-tuned sigmoid controller.
\end{abstract}

Key words: nonlinear feedback, sulfate-reducing process, continuous bioreactor, Lyapunov stability.

\section{Introduction}

Sulfate reduction is widely used for treating sulfate-containing wastewaters from industries, such as paper, textiles, pulp, and mining. In these processes, sulfate is converted to sulfide as the end product. Hence, it is ideally suited for treating metalcontaining wastewater from which heavy metals are simultaneously removed through the formation of metal sulfides. The current approach to biological treatment of complex toxic wastewater relies primarily on the use of microbial cultures. Many industrial waste treatment systems favor anaerobic processes because they usually require fewer resources [1].

On the other hand, the high demanding of the treatment regulations of industrial and municipal wastewaters have lead towards the addition of alternative unit processes, towards the renewal of the existing ones and towards the increased treatment chemical dosing and energy consumption in the wastewater treatment plants. This has made the cost-efficient operation of the plants more complicated. Besides, considerable development in instrumentation, automation and communication technologies has taken place during the recent decades, which has made possible the use of more advanced process control solutions in this kind of processes.

Process control aims are to increase the operation efficiency of the plants, therefore advanced process control algorithms can be used for the more accurate control of the process variables in comparison with the traditional operational methods. For example, one can estimate the influence of a control action on the process performance in the near future using mathematical models. An accurate mathematic process model developed using the process understanding and the operation data of a plant is needed for model predictive control. Process control is applied at several levels, such as the unit process level and plant-wide level.

The bioreactors processing is in most cases full of operational problems concerning essentially in the form of input and output multiplicities [2]. When input multiplicities occur, there is always the possibility of a transition from one steady state to another without detecting it; moreover, input multiplicity implies the existence of operational zones where the uncontrolled dynamics of the process could be unstable.

Despite the highly nonlinear dynamic of the bioreactor behavior, it is generally controlled via classical linear control approaches as classical proportional-integral-derivative (PID) schemes; the capacity of PID controllers to regulate most practical processes has led to their wide acceptance in industrial applications [3, 4], where these controllers perform well for processes with smooth dynamics and low performance requirements in narrow operating regions.

A detailed review of control strategies and their corresponding application can be found in the works of [5] and [6]. Some methods related to system model linearization as well as feedback linearization have also been considered [7]. Aguilar et al. [8] used an observer-based feedback linearized controller to regulate the bioreactor operation leading the substrate concentration to a desired set-point. Ramaswamy et al. [9] used model predictive control (MPC) to steer a continuous stirred tank reactor output towards a required set-point. Moreover, MPC have been employed for system with linear and smooth dynamic; therefore, MPC does not always address the nonlinear nature of systems, as the technique needs empirical and linearized system representations, making possible a poor system performance for even well-designed MPCs [9]. Bakosova et al. [10], propose a linear robust static output feedback con-

*e-mail: raguilar@ cinvestav.mx 
R. Aguilar-López and I. Neria-González

troller to regulate the nonlinear system with parametric uncertainties. Furthermore, in [11, 12], the authors have demonstrated theoretically and experimentally the superior performance nonlinear controller over conventional PI controller for bioreactor with input multiplicities. From the above, some nonlinear controllers have been proposed for bioreactor's control, considering adaptive, neural networks and robust frameworks [12-14], in particular for anaerobic sulfate reducing bioreactors $[15,16]$.

From the above, in this work it is proposed a class of nonlinear controller which allows regulating the dynamic behavior of a continuous stirred tank bioreactor, where the sulfate mass concentration is controlled via sulfate concentration measurements.

\section{Bioreactor modeling}

For sulfate-reducing bacteria (SRB) has been reported the inhibitory effect of sulfide in form of $\mathrm{H}_{2} \mathrm{~S}$ on cell growth in both batch and continuous cultures. Depending on the species of the SRB have been reported inhibitory concentrations of $\mathrm{H}_{2} \mathrm{~S}$ in an interval of 250 to $550 \mathrm{mgL}^{-1}$ [17].

According to the nature of sulfate-reducing process, the kinetic of growth of SRB is limited by the main products (i.e. hydrogen sulfide and acetate) related to oxidation of substrates as sulfate and lactate [18]. Considering this, for kinetic modeling, the structure of the corresponding model should consider the concentrations of these products. An important aspect to consider in the application of these processes is related to selection or development of the mathematical model kinetic of growth of SRB.

In this work, the purpose is to approximate the kinetic of growth anaerobic sulfate-reducing bacterium classified as $D$. alaskensis 6SR, where is proposed a double substrate with product inhibition unstructured kinetic model in accordance with the following structure which belongs to kinetic model proposed by Keehyun and Levenspiel [19]:

$$
\mu\left(x_{2}, x_{3}, x_{4}\right)=\mu_{\max }\left(\frac{x_{2}}{k_{s 1}+x_{2}}\right)\left(\frac{x_{4}}{k_{s 2}+x_{4}}\right)\left(1-\frac{x_{3}}{P^{*}}\right)^{n},
$$

where the kinetic parameter's set is given as follows: $\mu_{\max }=$ $0.1682 \mathrm{~h}^{-1} ; k_{s 1}=2296 \mathrm{mg} \mathrm{L}^{-1} ; k_{s 2}=1387 \mathrm{mg} \mathrm{L}^{-1}$; $P *=613.5575 \mathrm{mg} \mathrm{L}^{-1} ; n=0.615$; and $x_{2}, x_{3}$ and $x_{4}$ are the sulfate, sulfide and lactate mass concentrations, respectively.

The kinetic model given by Eq. (1) was compared with experimental data sets and a correlation coefficient of $R^{2}=$ 0.971 was obtained, as reported in [20]. Now, from the above kinetic model for the specific cell growth rate, the following mass balances equations are generated, considering continuous operating mode:

Biomass $\left(x_{1}\right)$ mass balance:

$$
\frac{d x_{1}}{d t}=-D x_{1}+\mu\left(x_{2}, x_{3}, x_{4}\right) x_{1} .
$$

Sulfate $\left(x_{2}\right)$ mass balance:

$$
\frac{d x_{2}}{d t}=D\left(x_{2, \text { in }}-x_{2}\right)-\mu\left(x_{2}, x_{3}, x_{4}\right) x_{1} Y_{1} .
$$

Sulfide $\left(x_{3}\right)$ mass balance:

$$
\frac{d x_{3}}{d t}=-D x_{3}+\mu\left(x_{2}, x_{3}, x_{4}\right) x_{1} Y_{2} .
$$

Lactate $\left(x_{4}\right)$ mass balance:

$$
\frac{d x_{4}}{d t}=D\left(x_{4, \text { in }}-x_{4}\right)-\mu\left(x_{2}, x_{3}, x_{4}\right) x_{1} Y_{3} .
$$

Acetate $\left(x_{5}\right)$ mass balance:

$$
\frac{d x_{5}}{d t}=-D x_{5}+\mu\left(x_{2}, x_{3}, x_{4}\right) x_{1} Y_{4},
$$

where $Y_{1}=8.1355 ; Y_{2}=1.5885 ; Y_{3}=13.631 ; Y_{4}=$ 8.5139 , here $Y_{1}$ is the sulfate-biomass yield coefficient, $Y_{2}$ is the sulfide-biomass yield coefficient, $Y_{3}$ is the lactate-biomass yield coefficient and $Y_{4}$ is the acetate-biomass yield coefficient.

Here the $x_{2, i n}$ and $x_{4, \text { in }}$ are the inlet concentration of sulfate and lactate and the $D=F / V$ is the named dilution rate, where $F$ is the input/output volumetric flow rate and $V$ is the corresponding volume of the reacting mixture, which is assumed as a constant.

\section{Proposed controller}

In general the system (2)-(5) can be generally represented by the following nonlinear state space representation:

$$
\dot{x}=f(x(t))+g(x(t)) u(x(t))
$$

and measured output:

$$
y=h(x(t))=C x(t),
$$

here $x(t) \in \mathbb{R}^{n}$ is the state space vector, $f(x(t))$ is a nonlinear smooth vector field, $f(x(t)):=\left\{f: \mathbb{R}^{n} \rightarrow \mathbb{R}^{n}\right\}$, $g(x(t)):=\left\{g: \mathbb{R}^{n} \rightarrow \mathbb{R}^{n x m}\right\}$ is the coefficient matrix of the control input and $u(x(t)) \in R^{m}$ is the control input vector and $y \in \mathbb{R}^{q}$. Dimensions $n$ and $m$ correspond to number of states in the model and number of manipulate variables, $q$ is the number of output measures. Vector function $f(x(t))$ contains all nonlinear terms in the model and $g(x(t))$ matrix contains linear relationships with vector of manipulate variables $u(x(t))$ It is assumed that the state variables belong to a given compact set $\aleph_{x} \in \mathbb{R}^{n}$ and all the closed-loop trajectories with initial conditions $x(0)$ in $\aleph_{x}$ remain bounded.

Assumptions:

$A 1$. The smooth and nonlinear vector field $f(x(t))$ is bounded, i. e. $\|f(x(t))\| \leq F<\infty$.

$A 2$. The coefficient matrix of the control input $g(x(t))$ is bounded, i. e. $\|g(x(t))\| \leq \Sigma<\infty$.

Defining the regulation error as $\varepsilon(x(t))=y(t)-y_{s p}=$ $C\left(x(t)-x_{s p}\right)$ where $x_{s p}$ is a constant set point, the dynamic equation for the regulation error is given by:

$$
\dot{\varepsilon}=f(x(t))+g(x(t)) u(x(t)) .
$$

Therefore, inspired in the work of [21], the following controller is considered:

$$
u(x(t))=-k(\exp (\varepsilon(x(t)))-1) .
$$

The proposed structure given by Eq. (10) provides closedloop stability to the system (7). Note that for negative values 
of the regulation error, the control law (12) tends to the value of the control gain $k$ and for positive values of the regulation error the controller tends to negative bounded values, considering that the regulation error is also bounded by physical constraints; when regulation errors are close to zero, means that the controlled state variable is close to the set point value, the controller tends to zero and it is off. From the above, the considered control structure tries to compensate the nonlinearities of the system (7), imposing a stable closed-loop behaviour.

3.1. Closed-loop stability sketch of proof. Consider the closed-loop state equation given by Eq. (9) under the control law (10)

$$
\dot{\varepsilon}=f(x(t))-g(x(t))(k(\exp (\varepsilon(x(t)))-1)) .
$$

Now, in order to analyze with detail the closed-loop stability of the system (11), it is needed to solve Eq. (11), as observed it is a highly nonlinear ordinary differential equation where the analytical solution is difficult to obtain. However, considering the monotonic behavior of the exponential function in the proposed control input, it is considered the Lyapunov second method [22] and the well-known principle of LaSalle's as well as a well arranged mathematical model described in Sec. 2, in order to show the general stability characteristic of the closed-loop dynamic of the system (11). Proposing the following Lyapunov candidate function $L$ as a quadratic form of the regulation error:

$$
\mathcal{L}=\frac{1}{2} \varepsilon^{2}
$$

The derivative along the trajectories of (12) is:

$$
\dot{\mathcal{L}}=\varepsilon \dot{\varepsilon}=\varepsilon(x(t))(f(x(t))+g(x(t)) u(x(t))) .
$$

Maximizing Eq. (13):

$$
\dot{\mathcal{L}} \leq\|f(x(t))+g(x(t)) u(x(t))\|\|\varepsilon(x(t))\| .
$$

Then:

$$
\begin{gathered}
\dot{\mathcal{L}} \leq[\|f(x(t))\|-\|g(x(t))\| \\
\cdot\|k(\exp (\varepsilon(x(t)))-1)\|]\|\varepsilon(x(t))\| .
\end{gathered}
$$

Applying assumptions $A 1$ and $A 2$ :

$$
\dot{\mathcal{L}} \leq[\mathcal{F}-k \Sigma\|(\exp (\varepsilon(x(t)))-1)\|]\|\varepsilon(x(t))\| .
$$

Considering that:

$$
0 \leq\|(\exp (\varepsilon(x(t)))-1)\| .
$$

For $\varepsilon(x(t)) \neq 0$.

It is required that:

$$
\mathcal{F}<k \Sigma \text {. }
$$

Then:

$$
\mathcal{F}-k \Sigma<0
$$

If $k>\mathcal{F}(\Sigma)^{-1}$; then:

$$
\dot{\mathcal{L}}<0
$$

Selecting adequately the control's gain $k$, by consider the equation criteria (18) and under the Lyapunov theory framework this provides semi-global and closed-loop stability for the system (11).

Considering an alternative representation of the Lyapunov function time-derivative as:

$$
\dot{\mathcal{L}}=\nabla \mathcal{L} \dot{\varepsilon}
$$

where $\nabla \mathcal{L}$ is defined as the gradient of the Lyapunov function $\mathcal{L}$ along the trajectory of the regulation error vector $\varepsilon(x(t))$. As it is known, the time-derivative of the Lyapunov function must be negative-definite is order to assure asymptotic stability of the system (11), therefore the corresponding inner product of the gradient and the tangent vector $\dot{\varepsilon}$ must be defined constantly negative, such that the angle between the corresponding vector must be larger than $90^{\circ}$, so that the Lyapunov function surface $\mathcal{L}$ is monotonically decreasing to zero. In consequence the system trajectory of the regulation error $\varepsilon(x(t))$, and the projection on the corresponding domain, converges to the origin as the time evolves.

Now, the trivial case, when $\varepsilon(x(t))=0$.

Then:

$$
\dot{\mathcal{L}}=0
$$

Finally, considering the both two mentioned possibilities:

$$
\dot{\mathcal{L}} \leq 0
$$

Therefore, from the Lyapunov second method, the system (11) is closed-loop stable.

Now, considering the application to the continuous bioreactor, it is represented by the following Single Input - Single Output (SISO) non-linear system from the sulfate $\left(x_{2}\right)$ mass balance (Eq. (3)), which is assumed as the controlled state equation:

$$
\dot{x}_{2}=\mu\left(x_{2}, x_{3}, x_{4}\right) x_{1} Y_{1}+\left(x_{2, i n}-x_{2}\right) D,
$$

where the system measured output is also the sulfate concentration:

$$
y=x_{2} .
$$

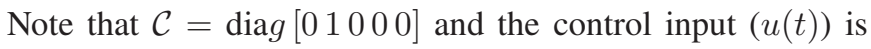
selected as the dilution rate:

$$
u(x(t))=D .
$$

The assumptions $A 1$ and $A 2$ for our particular case are:

$$
\begin{gathered}
\left\|\mu\left(x_{2}, x_{3}, x_{4}\right) x_{1} Y_{1}\right\| \leq \mu_{\max }=\mathcal{F}<\infty, \\
\left\|\left(x_{2, \text { in }}-x_{2}\right)\right\| \leq x_{2, \text { in }}=\Sigma<\infty .
\end{gathered}
$$

Note that the bounds of the both above terms are giving by physical constraints related with chemical kinetic and mass balance principles.

The regulation error dynamics in term of the original state space variables is giving as follows:

$\dot{\varepsilon}=\mu\left(x_{2}, x_{3}, x_{4}\right) x_{1} Y_{1}-\left(x_{2, i n}-x_{2}\right)(k(\exp (\varepsilon(x(t))-1))$. 
Now the domain of the state space bioreactor model is the set $\Omega \subset \Re^{5}$, therefore;

$$
\Omega=\left\{\begin{array}{l}
\left(x_{1}, x_{2}, x_{3}, x_{4}, x_{5}\right) \in \Re_{+}^{5} / 0 \leq x_{1} \leq x_{1, \max } ; \\
0 \leq x_{2} \leq x_{2, i n ;} ; 0 \leq x_{3} \leq x_{3, \max } ; \\
0 \leq x_{4} \leq x_{4, \text { in }} ; 0 \leq x_{5} \leq x_{5, \max }
\end{array}\right\} .
$$

Remember that the sub-index in is refereed as input bioreactor concentrations and the sub-index max is refereed as the maximum reached concentrations allowed by kinetic and thermodynamics constraints.

\section{Numerical results}

Numerical simulations were carried out in order to show the open-loop and closed-loop behavior of the anaerobic bioreactor. A PC computer with Intel Core 17 processor and ode $23 \mathrm{~s}$ library from MatLab to solve the ordinary differential equations system were employed; the initial conditions for the mass concentrations were $x_{10}=125 \mathrm{mg} / \mathrm{L}, x_{20}=5250 \mathrm{mg} / \mathrm{L}$, $x_{30}=50 \mathrm{mg} / \mathrm{L}, x_{40}=2750 \mathrm{mg} / \mathrm{L}$ and $x_{50}=25 \mathrm{mg} / \mathrm{L}$. Figures 1 and 2 are related with the open-loop behavior of the mass concentrations of all the state variables, it is observed a relatively high value of the sulfate concentration, around $2800 \mathrm{mg} / \mathrm{L}$ at steady state condition, this is an indicator of a poor performance of the bioreactor for sulfate removal purposes when the dilution rate is $D=0.025$ hours $^{-1}$. Figure 3 shows the corresponding phase portrait for the sulfate reducing process, where stable trajectories are showed; Fig. 4 presents the phase portrait of the carbon source consumption with a stable trajectory too. The closed-loop behavior of the continuous bioreactor in Figs. 5 and 6 where the proposed controller is turned-on at time $t=150$ hours, considering that the sulfate concentration is the measured and controlled state variable, the consuming sulfate concentration in the medium can be measured by turbid metric method based on the pre- cipitation of barium, which is a fast and simple method [22]; the control's gain is selected as $k=10$ hours $^{-1}$, in order to show the controller's capabilities two set points for the sulfate concentrations are selected to increase and diminish the sulfate concentration in comparison with the corresponding open-loop behavior. Firstly, the sulfate concentration is forced to reach a set point of $3000 \mathrm{mg} / \mathrm{L}$ with a setting time of the order of 30 hours without overshoots in this dynamic behavior, furthermore, to show, via numerical simulations, the capability of the proposed methodology at time $t=250$ hours, the required set point is changed to $2725 \mathrm{mg} / \mathrm{L}$, again the performance of the bioreactor is satisfactory and the new set point is reached without difficulties; note that all the uncontrolled mass concentrations (zero dynamic) remain with stable dynamic closed-loop response. On other hand, Fig. 7 shows the performance of the closed-loop trajectories of the sulfatereducing process under different initial conditions, it is observed that both two trajectories reach the required set point. Figure 8 shows the performance of the control effort given by the dynamic behavior of the dilution rate, the control input has an open-loop nominal value of 0.025 hours $^{-1}$, when the controller is turned-on the dilution rate is moved to around 0.05 hours $^{-1}$ to reach a steady state of 0.033 hours $^{-1}$ in a smooth way for the first considered set point, when the set point is changed to $2750 \mathrm{mg} / \mathrm{L}$ the dilution rate is diminished close to zero and then, a second steady state is reached with $D=0.023$ hours $^{-1}$. It is important to note that the required effort of the proposed controller belongs to physically realizable domain. Finally, a comparison with a class of sigmoid controller [16] under the same control's gain was done via a performance index named Integral Time-weighted Square Error (ITSE) which more penalize large control errors at long times, Fig. 9 shows the better performance of the proposed methodology.

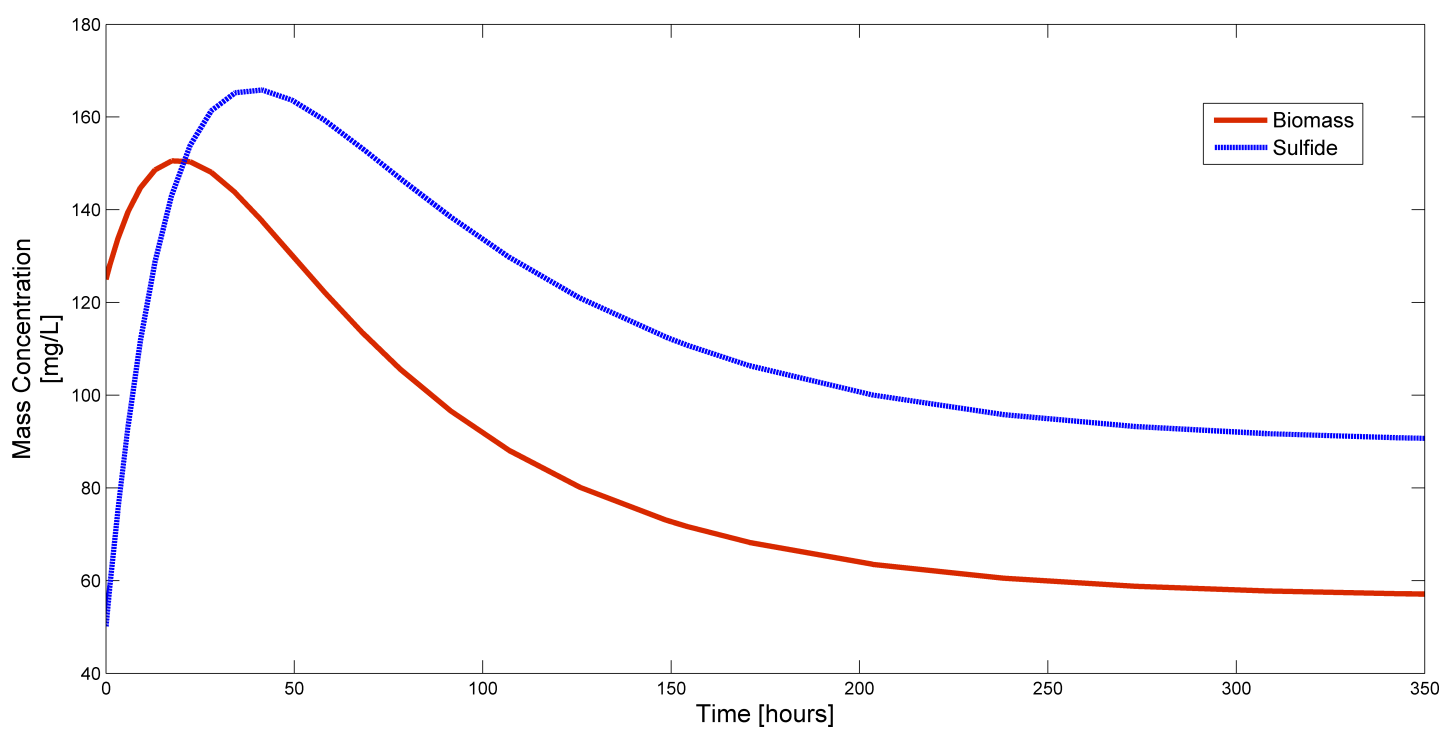

Fig. 1. Open-loop dynamics of Biomass and Sulfide Concentrations 
Controlling continuous bioreactor via nonlinear feedback: modelling and simulations approach

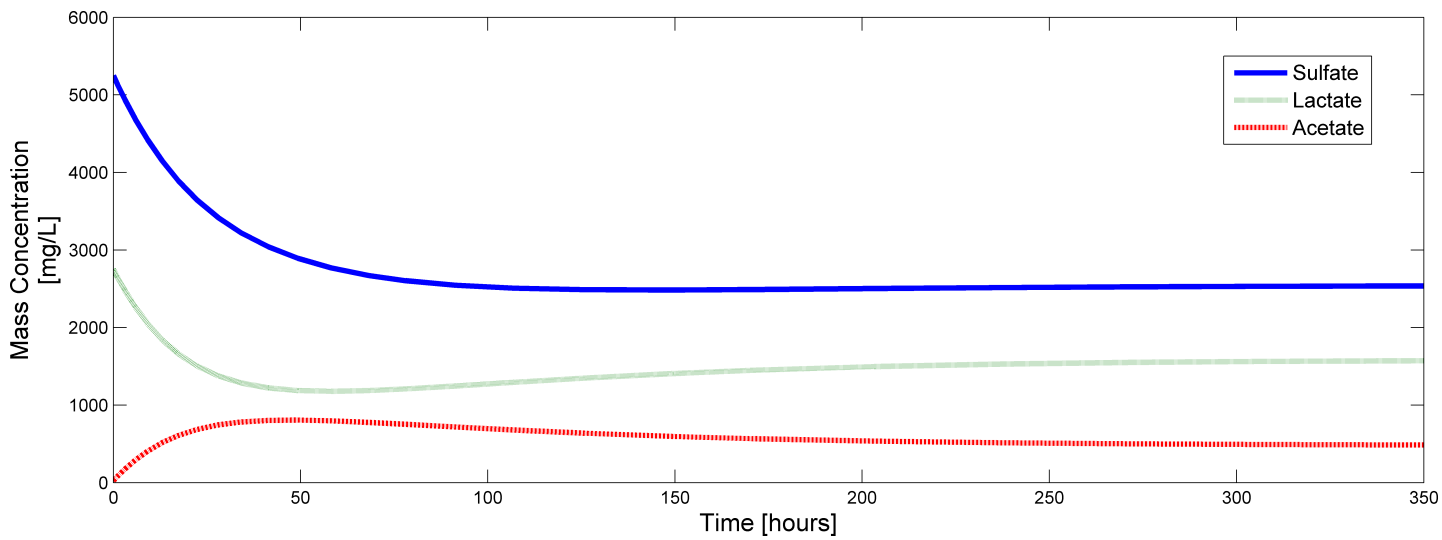

Fig. 2. Open-loop dynamics of Sulfate, Lactate and Acetate Concentrations

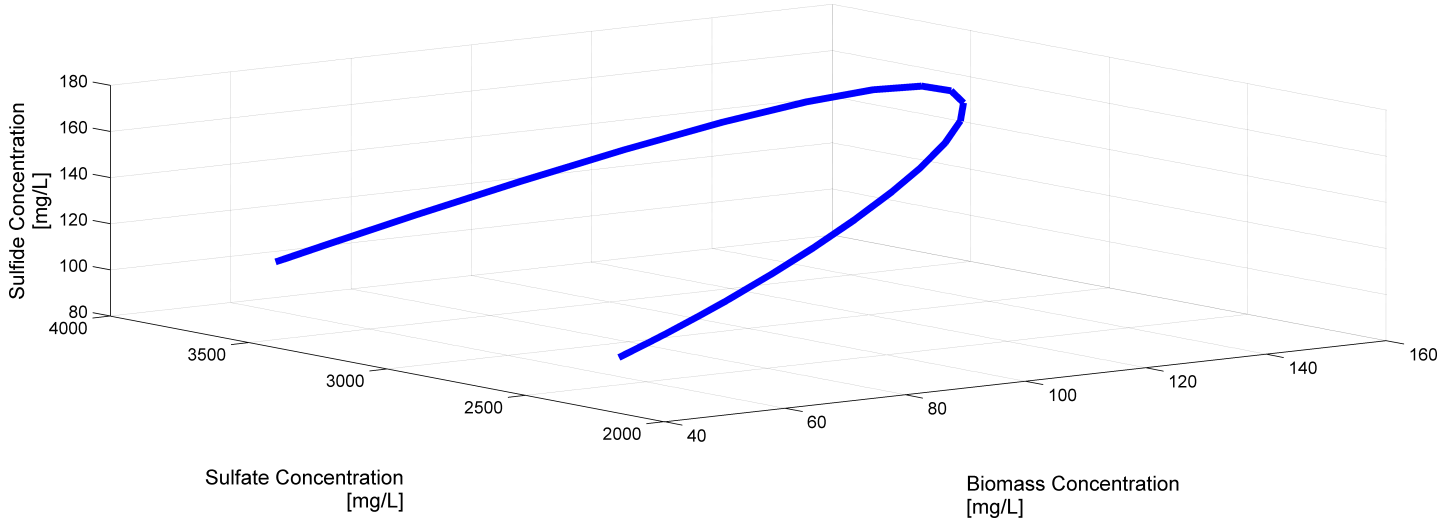

Fig. 3. Open-loop phase portrait of the sulfate-reducing process

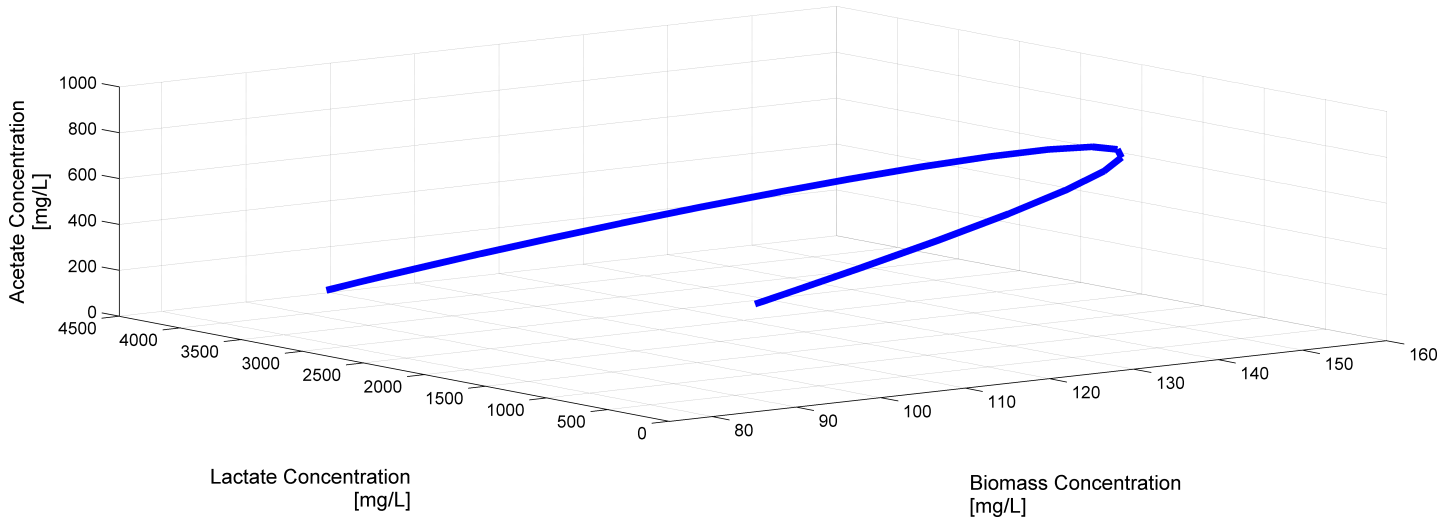

Fig. 4. Open-loop phase portrait of the carbon source consumption process

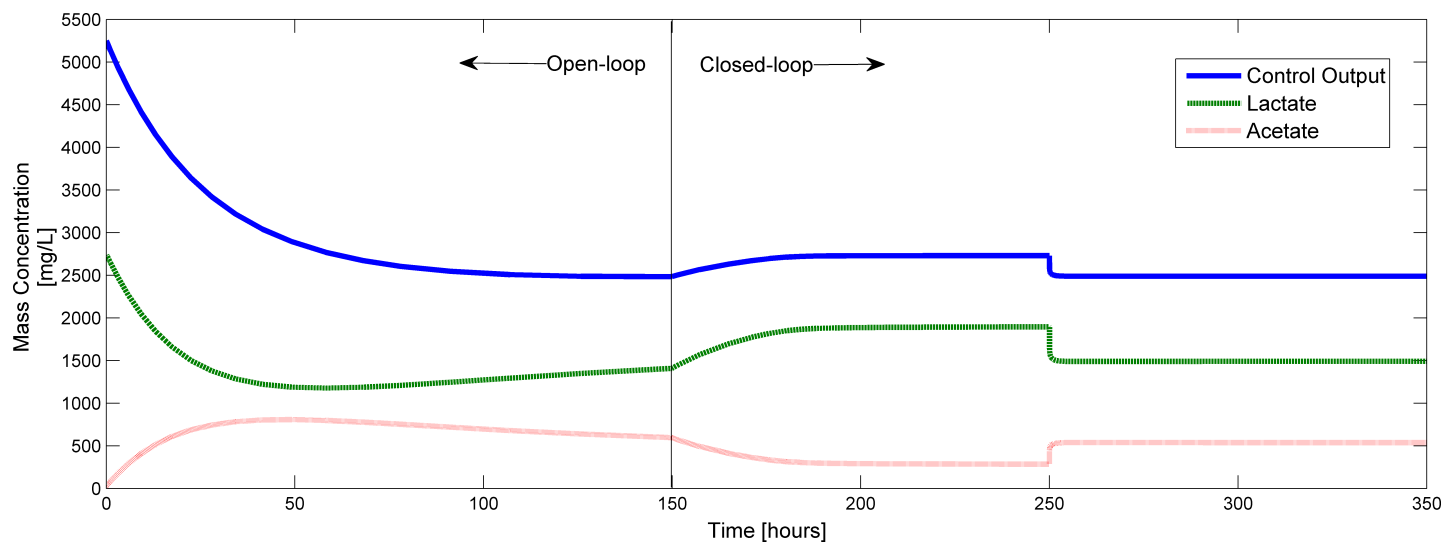

Fig. 5. Closed-loop dynamics 
R. Aguilar-López and I. Neria-González

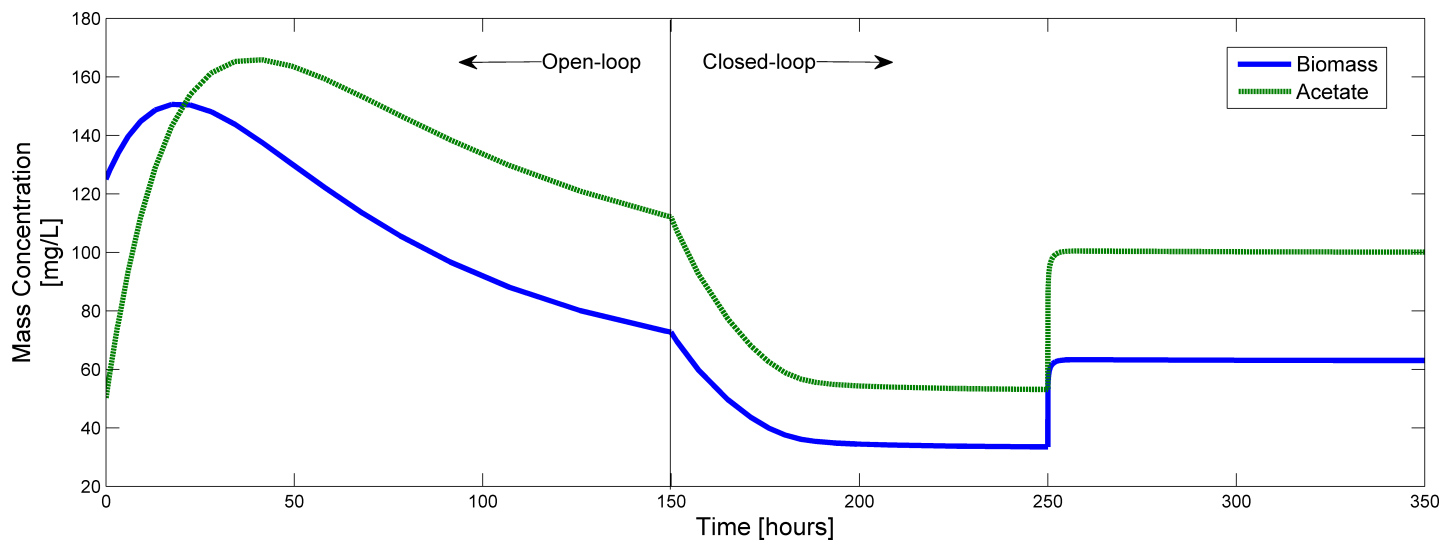

Fig. 6. Closed-loop dynamics

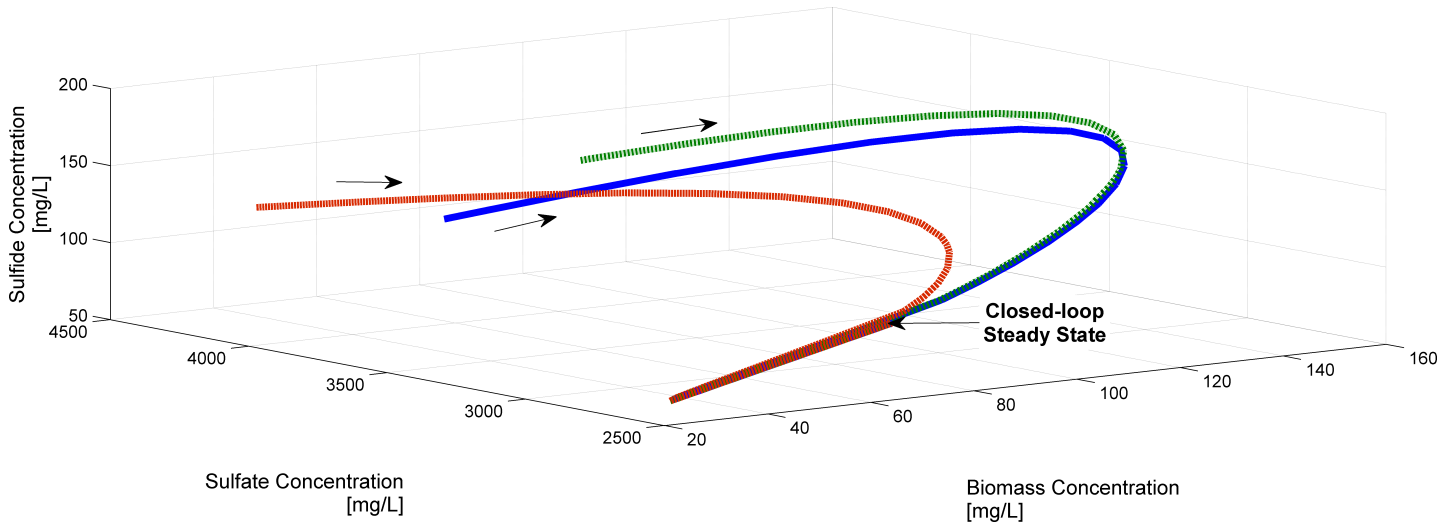

Fig. 7. Closed-loop phase portrait of the sulfate-reducing process under different initial conditions

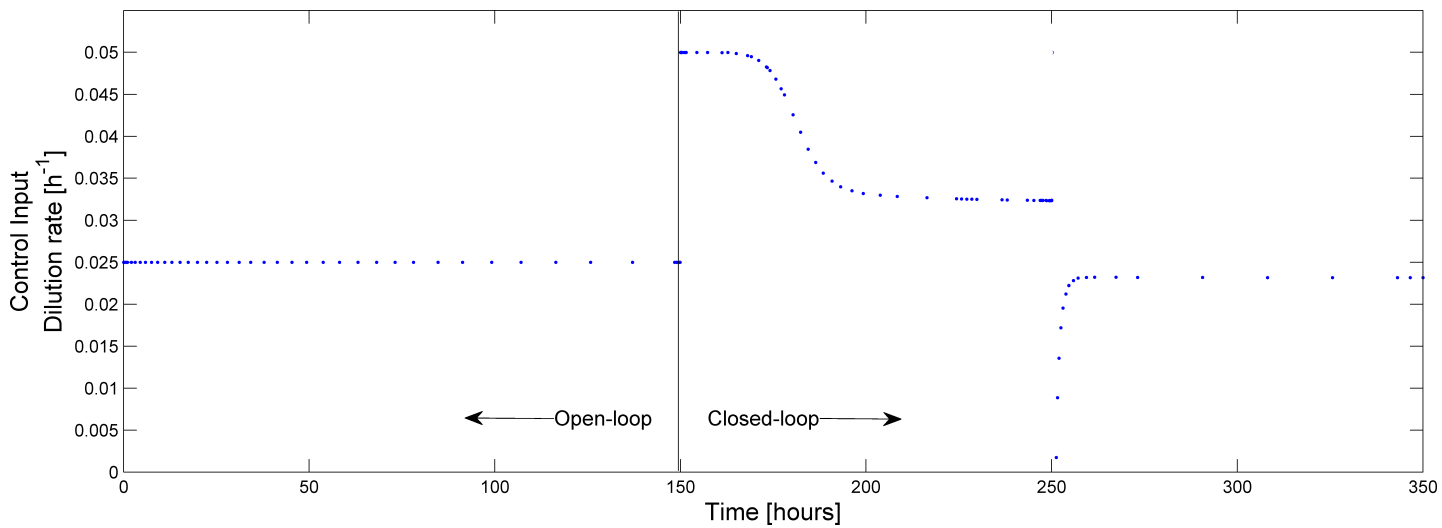

Fig. 8. Control effort

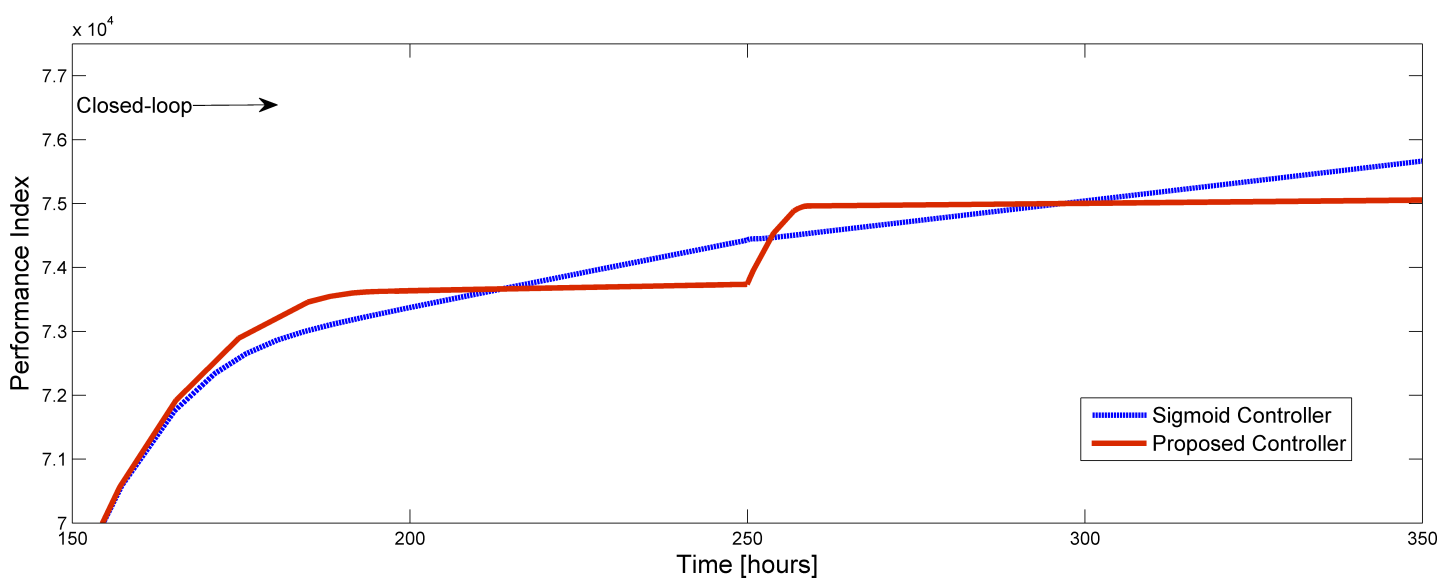

Fig. 9. Dynamic behavior of the performance index 
Controlling continuous bioreactor via nonlinear feedback: modelling and simulations approach

\section{Conclusions}

In this work a kinetic model experimentally corroborated for a sulfate-reducing process is presented, this model is employed as a benchmark on a continuous stirred tank bioreactor, where an exponential-type feedback is considered to regulated the sulfate concentration in the bioreactor via sulfate concentration measurements manipulating the dilution rate (input flow). The proposed controller is able to lead to the sulfate concentration to the required set points, with a satisfactory effort as showed by the numerical simulations, in agreement with the closed-loop stability analysis. For comparison purposes a sigmoid controller is implemented and the proposed one shows a better performance.

\section{REFERENCES}

[1] P.A. López Pérez, R. Aguilar López, and M.I. Neria González, "Cadmium removal at high concentration in aqueous medium: mediated by Desulfovibrioalaskensis", Int. J. Env. Sci. and Tech., DOI: 10.1007/s13762-014-0601-4 (2015).

[2] L.A. Melo-Varela, S. Casanova-Trujillo, and G. Olivar-Tost, "Dynamics of a bioreactor with a bacteria piecewise-linear growth model in a methane-producing process", Math. Prob. in Engin. ID 685452, DOI.org/10.1155/2013/685452 (2013).

[3] J. Alvarez-Ramirez and J. Alvarez, "PI regulation for a class of bioreactors: stability and performance", Int. J. Rob. and Nonlinear Ctl. 24 (5), 918-929 (2014).

[4] R. Aguilar, J. González, M.A. Barrón, R. Martínez, and R. Maya-Yescas, "Robust $\mathrm{PI}^{2}$ controller for continuous bioreactors", Proc. Biochem. 36 (10), 1007-1014 (2001).

[5] I.Y. Smets, J.E. Claes, E.J. November, G.P. Bastin, and J.F. Van Impe, "Optimal adaptive control of (bio)chemical reactors: past, present and future", J. Proc. Ctl. 14, 795-805 (2004).

[6] F. Angulo, R. Munoz, and G. Olivar, "Control of a bioreactor using feedback linearization", Proc. Medit. Conf. on Ctl. Auto. 1, 1-6 (2007).

[7] M.I. Neria.González, R. Martínez-Guerra, and R. AguilarLópez, "Feedback regulation of an industrial aerobic wastewater plan", Chem. Eng. J. 139, 475-481 (2008).

[8] S. Ramaswamy, T. Cutright, and H. Qammar, "Control of a continuous bioreactor using model predictive control", Proc. Biochem. 40 (8), 2763-2770 (2005).
[9] M. Bakosova, D. Puna, and A. Meszarosa, "Control of a continuous time stirred tank reactor via robust static output feedback", Proc. Medit. Conf. on Ctl and Aut. 1, 1-6 (2006).

[10] S.V. Sunil Kumar, V. Ramesh Kumar, and G. Prabhaker Reddy, "Nonlinear control of bioreactors with input multiplicities- an experimental work", Bioproc. and Biosyst. Eng. 28 (9), 45-53 (2005).

[11] J. Boskovic and K.S. Narendra, "Comparison of linear, nonlinear and neural-network based adaptive controllers for a class of fed-batch fermentation processes", Automatica 31 (6), 817-840 (1995).

[12] J. Ming-Feng, C. Yuh-Jongn, and C. Yi-Shyong, "Robust adaptive controller for continuous bioreactors", Biochem. Eng. J. 81, 136-145 (2013).

[13] D. Coutinho and A. VandeWouwer, "A robust non-linear feedback control strategy for a class of bioprocesses", IET Ctl. Theory and Appl. 7 (6), 829-841 (2013).

[14] P.A. López-Pérez, M.I. Neria-González, and R. Aguilar-López, "Nonlinear controller design with application to a continuous bioreactor", Theo. Found. of Chem. Eng. 47 (5), 585-592 (2013).

[15] P.A. López-Pérez, M.I. Neria-González, and R. Aguilar-López, "Cadmium concentration stabilization in a continuous sulfate reducing bioreactor via sulfide concentration control", Chem. Pap. 67 (3), 326-335 (2013).

[16] M. Reis, J. Lemos, and M. Carrondo, "Effect of hydrogensulfide on growth of sulfate reducing bacteria", Biotech. and Bioeng. 40 (5), 593-600 (1992).

[17] M. Reis, P.C. Lemos, J.S. Almeida, and M. Carrondo, "Influence of produced acetic-acid on growth of sulfate reducing bacteria”, Biotech. Lett. 12 (2), 145-148 (1990).

[18] H. Keehyun and O. Levenspiel, "Extended Monod kinetic for substrate, product, and cell inhibition", Biotech. and Bioeng. 32, 430-437 (1987).

[19] V. Peña Caballero, "Analysis of the operation of hybrid processes for the removal of $\mathrm{Cr}(\mathrm{VI})$ ", $P h$ D Thesis, CINVESTAV-IPN, México, 2013, (in Spanish).

[20] P. Luoudop, H. Fotsin, E.B. Megam Nguonkadi, S. Bowong, and H.A. Cerdeira, "Effective synchronization of a class of chua's chaotic systems using an exponential feedback coupling", Abs. and App. Anal. 20 ID 483269, 7 (2013).

[21] H.K. Khalil, Nonlinear Systems, Prentice Hall, New York, 1996

[22] A. Kolmer, P. Wikström, and K. Hallberg, "A fast and simple turbidimetric method for the determination of sulfate in sulfatereducing bacteria cultures", J. Microbio. Meth. 41, 179-184 (2003). 